\title{
New Additive Wavelet Image Fusion Algorithm for Satellite Images
}

\author{
B. Sathya Bama*, S.G. Siva Sankari, R. Evangeline Jenita Kamalam, \\ and P. Santhosh Kumar \\ Thigarajar College of Engineering, Department of ECE, Madurai, Tamil Nadu, India \\ \{sbece, sivasankari, evangelinejenita\}@tce.edu rsanfun@gmail.com
}

\begin{abstract}
Fusion of Low Resolution Multi Spectral (LRM) image and High Resolution Panchromatic (HRP) image is a very important topic in the field of remote sensing. This paper represents an efficient method for image fusion using New Additive Wavelet transform (NAW) based on the à trous algorithm. The fused image should preserve both geometric and the radiometric information. The use of geometric features along with spectral information improves the visualization quality of imagery. In this method intensity of the LRM image is added to the difference of the wavelet planes of HRP and LRP. The experimental results show that this method can well preserve spectral and spatial details of the source images. The proposed method provides competitive or even superior results for the input images compared to other well-known methods by providing $85.25 \%$ Quality with No Reference (QNR).
\end{abstract}

Keywords: correlation coefficient, discrepancy, MSSIM, radiometric distortion index, sobel grades, SSIM.

\section{Introduction}

Recent advancements in the field of remote sensing explore the opportunity of using images of different sensors for the same application. Pan sharpening is a kind of satellite image fusion method which injects the high-frequency components taken from the Pan image into resampled versions of the MS data to get a color image with pleasing and sharp appearance. Remote sensing calls for advanced pan sharpening methods to translate the spectral information of the coarse-scale MS data to the fine scale Pan image with minimal introduction of spectral distortions. Various methods have been proposed for pan-sharpening, which includes Intensity Hue Saturation (IHS) [1], the Principal Component Analysis and Multiresolution Transforms. The existing waveletbased image fusion methods are mostly based on two computation algorithms: the Mallat algorithm and the à trous algorithm [2]. The paper concentrates on the à trousalgorithm.

Generally, the à trous-algorithm-based image fusion can be performed in two ways: 1) Substitute-Wavelet (SW) method and 2) Additive-Wavelet (AW) method [3]. The

\footnotetext{
* Corresponding author. 
AW method maintains the high frequency of the HRP image and low frequency of the LRM image and the SW method eliminates the low frequency of the LRM image. However, if the same high frequency is injected into every LRM image, the AW method can produce redundant high frequency. As the HRP and LRM images generally have different local radiometry, radiometric distortion can arise in fused images. Also, the SW method can lose some of the information of the LRM image during substitution process [4].These problems can generate artifacts in fused image and it will suffer from radiometric and geometric distortions. To cope up with the above problem, this paper proposes a New Additive Wavelet algorithm based on à trous method. This paper is organized as follows section 2 describes the proposed method; section 3 discusses about the experimental results and section 4 conclude this paper.

\section{New Additive Wavelet Transform}

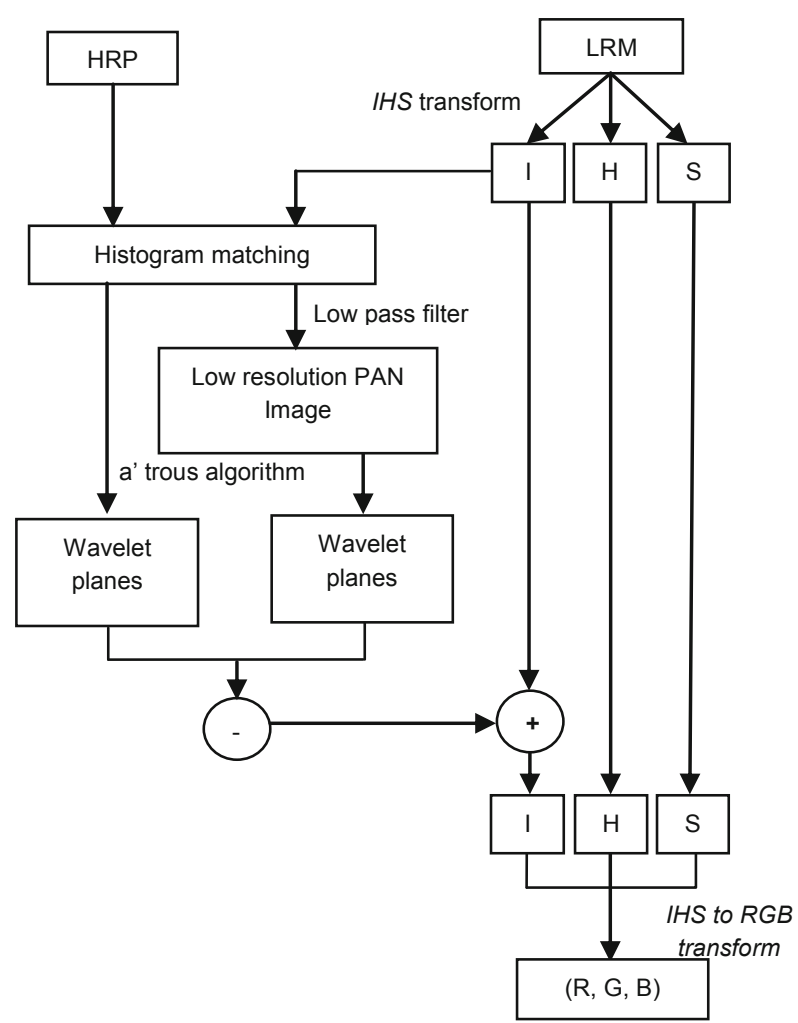

Fig. 1. New Additive Wavelet Transform 
Fig 1 shows the proposed à trous based fusion method to decompose the image into wavelet planes .The New Additive Wavelet transform can be formulated by the following procedure.

1. Transform the RGB images into the IHS color space.

2. Perform histogram matching between the HRP image and the intensity.

3. Decompose the matched HRP image to $n$ wavelet planes.

$$
H R P=\sum_{l=1}^{n} w_{H R P}+H R P^{r}
$$

For an image a sequence of approximated images are obtained by convoluting with the filter that has B3 cubic spline profile. It is followed by the computation of the wavelet planes by the difference between two consecutive approximations.

4. Decompose the LRP image to $n$ wavelet planes.

$$
L R P=\sum_{l=1}^{n} w w_{l}+L R P^{r}
$$

The LRP image is spatially degraded by filtering with a Gaussian low-pass filter.

5. Intensity of LRM image is added with the difference of wavelet planes of HRP and LRP images.

$$
I_{n e w}=I+\sum_{l=1}^{n} w H R P_{l}-\sum_{l=1}^{n} w L R P_{l}
$$

6. Transforming the new intensity together with the hue and saturation components back into RGB (inverse IHS transform).

The proposed method considers a bandpass injection of high frequency extracted from an HRP image instead of conventional high-pass injection. Subsequently, the difference between the high frequency of the HRP image scale and the low frequency of the LRP image scale is injected. Thus, the low frequency of the LRP image scale is discarded. This implies that the high frequency between the LRM and HRP images is injected while maintaining the LRM image. Adding the wavelet planes to the intensity component preserves the spectral quantity more than that adding to the RGB of the LRM image individually. Since spectral values are present only in Hue and Saturation component, adding these planes to the intensity component does not affect the spectral values.

\section{$3 \quad$ Data Sets and Experimental Results}

Experiments have been conducted to evaluate the performance of the proposed method using the WORLDVIEW-2 data of Madurai, Tamil Nadu, India. For all data sets, the LRM image size is $128 \times 128$, and the HRP image size is $512 \times 512$ pixels. The 
experiment area constitutes trees, buildings, and roads. The NAW method is compared with the existing methods.Fig. 2 shows the HRP image of resolution $0.46 \mathrm{~m}$. Fig. 3 is $1.84 \mathrm{~m}$ LRM image interpolated at $0.46 \mathrm{~m}$.Fig 4 shows AWLP fusion method. The image obtained by NAW method is shown in Fig.5. This method produces highquality image, implying that this image contains the necessary geometric information as well as rich radiometric information.

\subsection{Quantitative Analysis}

In Table 1, the reconstructed HR multispectral images are compared with the results produced by the existing methods such as IHS, wavelet, FFT enhanced IHS, Additive Wavelet (AW), Substitute Wavelet (SW). Table 1 demonstrates that the NAW the

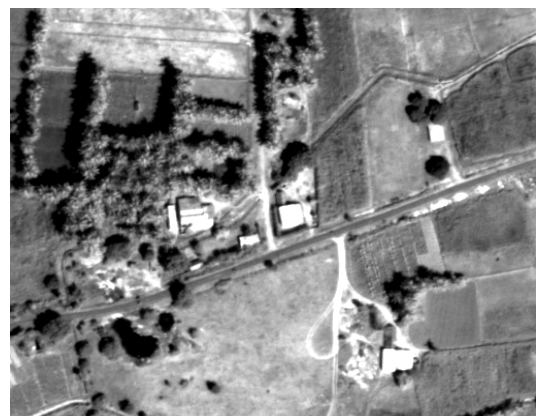

Fig. 2. HRP image

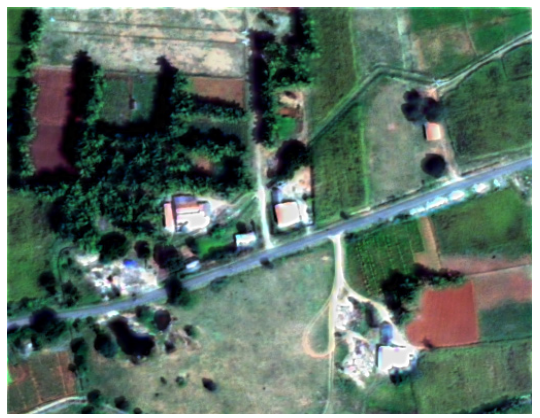

Fig. 4. AWLP fused image

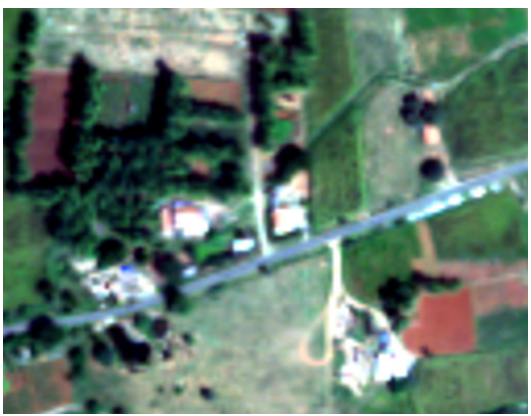

Fig. 3. LRM image

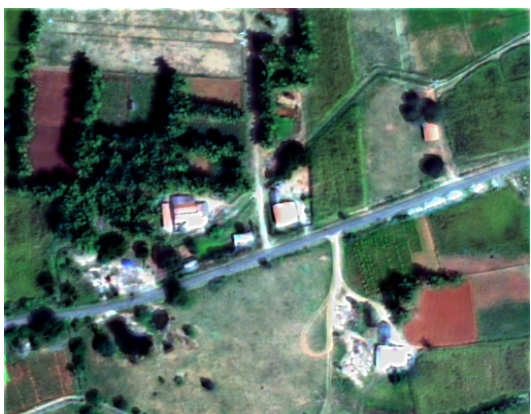

Fig. 5. NAW fused image

NAW method provides a less-distorted fused image compared with existing methods by finding Quality with No Reference (QNR) index [5]. In Table 2 shows the result of the spectral quality of the fused image measured by metrics such as discrepancy and Mean Structural-Similarity-Based Image Quality Assessment (MSSIM) [6].Spatial quality of the fused image is measured by metrics such as correlation coefficient [7] 
and sobel grade. NAW yields the smallest spectral difference i.e. discrepancy and highest MSSIM i.e. more similar spectral values of LRM image. From Table 1-2 it's known that NAW gives superior performance for most of the quality metrics.

Table 1. Quality Indexes Of The Fused Images

\begin{tabular}{|c|c|c|c|}
\hline Parameter & $\begin{array}{c}\text { Radiometric } \\
\text { distortion }\end{array}$ & $\begin{array}{c}\text { Geometric } \\
\text { distortion }\end{array}$ & QNR \\
\cline { 1 - 3 } IHS & $\mathbf{0 . 0 3 3 1}$ & $\mathbf{0 . 1 1 8 3}$ & $\mathbf{0 . 8 5 2 5}$ \\
\cline { 1 - 3 } $\begin{array}{c}\text { FFT En- } \\
\text { hanced IHS }\end{array}$ & $\mathbf{0 . 0 1 4 1}$ & $\mathbf{0 . 1 4 1 7}$ & $\mathbf{0 . 8 4 6 7}$ \\
\hline Wavelet & $\mathbf{0 . 0 2 3 1}$ & $\mathbf{0 . 1 3 4 1}$ & $\mathbf{0 . 8 4 5 8}$ \\
\hline AWLP & $\mathbf{0 . 0 5 4 9}$ & $\mathbf{0 . 1 2 2 0}$ & $\mathbf{0 . 8 2 9 7}$ \\
\hline SW & $\mathbf{0 . 0 3 2 6}$ & $\mathbf{0 . 1 2 0 7}$ & $\mathbf{0 . 8 5 0 6}$ \\
\hline NAW & $\mathbf{0 . 0 3 0 9}$ & $\mathbf{0 . 1 2 0 3}$ & $\mathbf{0 . 8 5 2 5}$ \\
\hline Ideal & $\mathbf{0}$ & $\mathbf{0}$ & 1 \\
\hline
\end{tabular}

Table 2. Spectral and spatial quality Indexes Of The Fused Images

\begin{tabular}{|c|c|c|c|c|c|c|c|c|c|c|c|c|}
\hline \multirow{2}{*}{$\begin{array}{c}\text { Parame- } \\
\text { ter }\end{array}$} & \multicolumn{4}{|c|}{ Spectral Quality } & \multicolumn{5}{c|}{ Spatial Quality } \\
\cline { 2 - 14 } & \multicolumn{3}{|c|}{ Discrepancy } & \multicolumn{3}{c|}{ MSSIM } & \multicolumn{3}{c|}{$\begin{array}{c}\text { Correlation } \\
\text { Coefficient }\end{array}$} & \multicolumn{3}{c|}{ Sobel Grade } \\
\hline Methods & R & G & R & G & R & G & R & G & B & R & G & B \\
\hline IHS & 11.96 & 17.66 & 16.65 & 0.16 & 0.21 & 0.41 & 0.87 & 0.96 & 0.90 & 42.5 & 52.1 & 46.3 \\
\hline $\begin{array}{c}\text { FFT- } \\
\text { Enhanced } \\
\text { IHS }\end{array}$ & 15.10 & 21.71 & 21.71 & 0.14 & 0.15 & 0.25 & 0.84 & 0.92 & 0.89 & 40.6 & 60.6 & 53.7 \\
\hline Wavelet & 13.43 & 17.43 & 15.43 & 0.17 & 0.20 & 0.34 & 0.89 & 0.96 & 0.91 & 40.1 & 40.8 & 37.9 \\
\hline AWLP & 22.20 & 30.13 & 22.23 & 0.18 & 0.19 & 0.32 & 0.85 & 0.93 & 0.88 & 40.8 & 54.8 & 52.7 \\
\hline Sw & 16.65 & 16.65 & 16.65 & 0.17 & 0.22 & 0.40 & 0.88 & 0.92 & 0.92 & 42.0 & 53.0 & 50.5 \\
\hline NAW & 11.83 & 15.70 & 14.04 & 0.18 & 0.25 & 0.42 & 0.89 & 0.97 & 0.92 & 48.6 & 60.6 & 55.7 \\
\hline
\end{tabular}

\section{Conclusion}

In this paper, New Additive Wavelet fusion method is proposed for preserving both radiometric information and geometric information. In this method bandpass injection of frequency by finding the difference between LRP and HRP image is done. The 
proposed method can be considered as an improvement of the SW and AW methods in the sense that the LRM image is not decomposed. The proposed method provides comparatively better results than other existing methods in terms of spectral and spatial quality metrics.

\section{References}

1. Choi, M.: A new intensity-hue-saturation fusion approach to image fusion with a tradeoff parameter. IEEE Trans. Geosci. Remote Sens. 44(6), 1672-1682 (2006)

2. González-Audícana, M., Otazu, X., Fors, O., Seco, A.: Comparison between the Mallat's and the 'à trous' discrete wavelet transform based algorithms for the fusion of multispectral and panchromatic images. Int. J. Remote Sens. 26(3), 597-616 (2005)

3. Núnez, J., Otazu, X., Fors, O., Prades, A., Pala, V., Arbiol, R.: Multiresolution-based image fusion with additive wavelet decomposition. IEEE Trans. Geosci. Remote Sens. 37(3), 1204-1211 (1999)

4. Stathaki, T.: Image Fusion Algorithms and Applications, 1st edn., ch. 6. Elsevier, Amsterdam (2008)

5. Alparone, L., Aiazzi, B., Baronti, S., Garzelli, A., Nencini, F., Selva, M.: Multispectral and panchromatic data fusion assignment without reference. Photogramm. Eng. Remote Sens. 74(2), 193-200 (2008)

6. Wang, Z., Bovik, A.C., Sheikh, H.R.: Image Quality Assessment: From Error Visibility to Structural Similarity. IEEE Transactions on Image Processing 13(4) (April 2004)

7. Zhou, J., Civico, D.L., Silander, J.A.: A wavelet transform method to merge landsat TM and SPOT panchromatic data. International Journal of Remote Sensing 19(4) (1998) 